\title{
MOORE'S PARADOX AND THE LOGIC OF BELIEF
}

\author{
ANDRÉS PÁEZ \\ https:/ / orcid.org/0000-0002-4602-7490 \\ Universidad de los Andes \\ Department of Philosophy \\ Bogotá-D.C. \\ Colombia \\ apaez@uniandes.edu.co
}

\section{Article info \\ CDD: 160 \\ Keywords \\ Moore's Paradox \\ Logic of Belief \\ Hintikka \\ Epistemic Logic}

Received: 15.06.2020; Accepted: 19.06.2020

https://doi.org/10.1590/0100-6045.2020.V43N2.AP

Abstract: Moore's Paradox is a test case for any formal theory of belief. In Knowledge and Belief, Hintikka developed a multimodal logic for statements that express sentences containing the epistemic notions of knowledge and belief. His account purports to offer an explanation of the paradox. In this paper I argue that Hintikka's interpretation of one of the doxastic operators is philosophically problematic and leads to an unnecessarily strong logical system. I offer a weaker alternative that captures in a more accurate way our logical intuitions about the notion of belief without sacrificing the possibility of providing an explanation for problematic cases such as Moore's Paradox. 


\section{INTRODUCTION}

Among the problems that any logical analysis of the notion of belief has to address, Moore's Paradox occupies a preeminent position. G. E. Moore observed that sentences such as 'It's raining but I don't believe it' cannot be used to make coherent assertions, even though they are not actual contradictions (1942, pp. 541-543; 1944, p. 204). There are situations in which the sentence will be true but none in which anybody could use it in a literal sense. In general, sentences of the form:

(1) ' $p$ but I do not believe that $p$ '

are not self-contradictory, but there are no circumstances in which one can use them to perform coherent assertoric speech acts. The divergence between the truth conditions and the performance conditions of (1) leads to the paradoxical result that there are true sentences that one cannot utter. ${ }^{1}$

That a sentence of this type is not self-contradictory is illustrated by the fact that a simple change of person turns (1) into the perfectly natural sentence

(2) ' $p$ but he does not believe that $p$ '.

\footnotetext{
${ }^{1}$ Moore's Paradox can be extended to other propositional attitudes (Searle 1969). For example, the statement 'Rain is likely, but I do not expect it' is also absurd. In this essay I will only be concerned with the notion of belief.
} 
Similarly, a change of tense also results in a coherent assertion:

(3) ' $p$ but I did not believe it'.

Finally, the absurdity of (1) also vanishes when the sentence is embedded in a larger context:

(4) 'Suppose that $p$ but I do not believe that $p$ '.

These characteristics of (1), which are not mirrored in the case of typical contradictions, seem to indicate that the problem is not a function of the truth conditions of the sentence, but rather of the performance conditions of the speech act that expresses it and, perhaps, of the mental analogue of these performance conditions for the corresponding propositional attitudes. For this reason, it has been argued that an adequate analysis of the notion of belief must be made in terms of statements, as opposed to sentences. Although an analysis in terms of sentences is all that is needed in most cases, there are certain properties of statements, such as the identity of the speaker and the recipient of his words, that cannot be defined solely in terms of the forms of words. On the other hand, an analysis in terms of sentences recommends itself due to the possibility of developing simplified logical systems which avoid the unmanageable task of defining complex performance conditions.

In Knowledge and Belief (1962), Hintikka tried to achieve the synthesis of both approaches. In that seminal work, Hintikka developed a multimodal logic for statements that express sentences containing the epistemic notions of knowledge and belief. Most of his analysis is made in terms of sentences, including his explanation of Moore's Paradox, but he describes the way in which the system can be 
expanded to handle sentences whose meaning varies according to the context of utterance and the identity of the speaker.

A conspicuous feature of Hintikka's analysis is that the logic of belief turns out to be parasitic on the logic of knowledge in the sense that the former is simply a weaker version of the latter. Although one would expect to find more than one similarity between both logics, the fact that the logic of belief is so closely modeled after the system developed for the notion of knowledge leads Hintikka to adopt a set of axioms that-I will argue-is unnecessarily strong and highly problematic. In this essay I will develop an alternative logical system for sentences containing the notion of belief. The system, which I will call $\mathrm{H}^{*}$, retains the basic elements of Hintikka's system but it is based on a weaker set of axioms. I will try to show that the axioms of $\mathrm{H}^{*}$ capture in a more accurate way our logical intuitions about the notion of belief without sacrificing the possibility of providing an explanation for problematic cases such as Moore's Paradox.

\section{THE SYSTEM H*}

Hintikka's analysis of the notion of belief is based on the two multimodal operators $B_{a}$ and $C_{a}$, which are the formal counterparts of ' $a$ believes that' and 'it is compatible with everything that $a$ believes that'. Each subscript $a, b, c, \ldots$ represents a different individual. Hintikka explains the intuitive idea behind the operator $C_{a}$ in terms of consistency. If my beliefs are consistent, it must be possible for all of them to turn out to be true without having to give up any of them. Similarly, if something is compatible with my consistent beliefs, then it must be possible for this something to turn out to be the case together with everything I believe without making it necessary for me to give up any of my beliefs (1962, 
p. 24). In formal terms, if the set $\left\{B_{a} q_{1}, B_{a} q_{2}, \ldots, B_{a} q_{k}, C_{a} p\right\}$ is consistent, then the set $\left\{B_{a} q_{1}, B_{a} q_{2}, \ldots, B_{a} q_{k}, q_{1}, q_{2}, \ldots, q_{k}, p\right\}$ must also be consistent.

This interpretation, however, is problematic. Clearly my beliefs are consistent if there is a possible state of affairs in which they are all true, but it is not obvious why it should be added that in that state of affairs I must possess those beliefs. If my beliefs are consistent, it is compatible with everything I believe that there is a state of affairs in which my beliefs are true even though I may not possess some of them. But Hintikka's interpretation of $C_{a}$ excludes that possibility because the set $\left\{B_{a} p, C_{a} \sim B_{a} p\right\}$ turns out to be inconsistent. ${ }^{2}$ On the other hand, if my beliefs are consistent, it is also compatible with everything I believe that there is a state of affairs in which my beliefs are true and I possess those beliefs. This is the only possibility that Hintikka allows.

Since there is no obvious reason to exclude the first possibility, my interpretation of $C_{a}$ will be as follows: If the set $\left\{B_{a} q_{1}, B_{a} q_{2}, \ldots, B_{a} q_{k}, C_{a} p\right\}$ is consistent, then the set $\left\{q_{1}\right.$, $\left.q_{2}, \ldots, q_{k}, p\right\}$ must also be consistent. Thus, according to this interpretation, the set $\left\{B_{a} p, C_{a} \sim B_{a} p\right\}$ will not be inconsistent. One of the challenges in developing the system $\mathrm{H}^{*}$ will be to formulate a set of axioms that capture these intuitions about the notion of belief.

The basis of $\mathrm{H}^{*}$ is as follows.

2 The reason is obvious. Suppose the set $\left\{B_{a} p, C_{a} \sim B_{a} p\right\}$ is consistent. Then, according to Hintikka's interpretation of $C_{a}$, the set $\left\{p, B_{a} p, \sim B_{a} p\right\}$ is consistent, which is absurd. 
Primitive Symbols

$$
\begin{aligned}
& p_{1}, p_{2}, p_{3}, \ldots, p_{k} \quad \text { [Propositional variables] } \\
& \sim, B_{a}, B_{b}, \ldots, B_{n}, C_{a} C_{b}, \ldots, C_{n} \quad \text { [Monadic operators] } \\
& \&, \vee, \supset, \equiv \quad \text { [Dyadic operators] }
\end{aligned}
$$

Formation Rules

FR1 A variable standing alone is a wff.

FR2 If $p$ is a wff, so is $\sim p$.

FR3 If $p$ and $q$ are wffs, and $\bullet$ is a dyadic operator, then ( $p$ - $q)$ is a wff.

FR4 If $p$ is a wff, then $B_{a} p$ and $C_{a} p$ are wffs.

Definitions:

[Def B] $\quad B_{a} p \equiv_{\text {Def }} \sim C_{a} \sim p$

[Def C] $\quad C_{a p} \equiv_{\text {Def }} \sim B_{a} \sim p$

\section{Axioms}

The theorems include all tautologies of the propositional calculus, plus the following axioms:

A1 $B_{a}(p \supset q) \supset\left(B_{a} p \supset B_{a} q\right)$

A2 $\quad B_{a p} \supset C_{a p}$

A3 $B_{a} p \supset C_{a} B_{a} p$

Transformation Rule

Modus Ponens (MP)

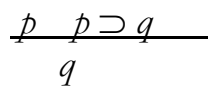

Using possible world semantics, we can provide an intuitively meaningful interpretation of the system. A model set is a partial description of a possible world. A set $\mu$ of sentences is a model set iff it satisfies the following conditions: 
(C. $\sim) \quad$ If $p \in \mu$, then $\sim p \notin \mu$.

(C.\&) $\quad$ If $p \& q \in \mu$, then $p \in \mu$ and $q \in \mu$.

(C. $\vee$ ) If $p \vee q \in \mu$, then $p \in \mu$ or $q \in \mu$ (or both).

(C. $\sim \sim$ ) If $\sim \sim p \in \mu$, then $p \in \mu$.

(C. $\sim \&$ ) If $\sim(p \& q) \in \mu$, then $\sim p \in \mu$ or $\sim q \in \mu$ (or both).

(C. $\sim \vee$ ) $\quad$ If $\sim(p \vee q) \in \mu$, then $\sim p \in \mu$ and $\sim q \in \mu$.

In order to provide a semantical interpretation of the multimodal operators $B_{a}$ and $C_{a}$, we need to make reference to more that one model set. The reason is obvious. If $p$ is compatible with my beliefs, then there must be at least one state of affairs in which $p$ turns out to be the case. But this state of affairs need not be identical with the one in which I believe that $p$. We will call a description of such state of affairs an alternative to $\mu$ with respect to $a$.

Let $\Omega$ be a set of model sets $\mu, \mu *, \mu * *, \ldots$ Such set of model sets will be called a model system. The following conditions must be imposed on a model set $\mu$.

(C.B) If $B_{a} p \in \mu$ and if $\mu$ belongs to a model system $\Omega$, then there is in $\Omega$ at least one alternative $\mu *$ to $\mu$ such that $p \in \mu^{*}$.

(C.B $\left.{ }^{*}\right) \quad$ If $B_{a} p \in \mu$ and if $\mu *$ is an alternative to $\mu$ in some model system $\Omega$, then $p \in \mu *$.

(C.C) If $C_{a} p \in \mu$ and if $\mu$ belongs to a model system

$\Omega$, then there is in $\Omega$ at least one alternative $\mu *$ to $\mu$ such that $p \in \mu *$. 
(C. CB) If $B_{a} p \in \mu$ and if $\mu$ belongs to a model system $\Omega$, then there is in $\Omega$ at least one alternative $\mu *$ to $\mu$ such that $B_{a} p \in \mu *$.

(C. $\left.B_{\text {Def }}\right) \quad B_{a} p \in \mu$ if and only if $\sim C_{a} \sim p \in \mu$

(C.C $\left.C_{\text {Def }}\right) \quad C_{a} p \in \mu$ if and only if $\sim B_{a} \sim p \in \mu$.

There are several important differences between the system $\mathrm{H}^{*}$ and the system proposed by Hintikka. Instead of axiom A3, Hintikka includes the following axiom in his system:

$$
B_{a} \supset B_{a} B_{a} p .
$$

In defense of (5), he argues (p. 25) that the axiom is necessary to prove that the following sentence is a contradiction:

(6) $\quad B_{a} p \&\left(B_{a} p \supset B_{a} \sim B_{a} p\right)$.

Although (6) is certainly contradictory, it is also true that our system, whose set of axioms is weaker than the set of axioms in Hintikka's system, suffices to show that it is. Consider the following reductio of (6) in our system:

(6) Bap \& (Bap $\supset$ Ba Bap) $\in \mu \quad$ Counterassumption

(7) Bap $\supset$ Ba Bap $\in \mu \quad$ From (6) by (C.\&)

(8) Bap $\in \mu \quad$ From (6) by (C.\&)

(9) Ba Bap $\in \mu \quad$ From (7) and (8) by Modus Ponens

(10) Bap $\in \mu^{*}$

From (8) by (C.CB)

(11) $\sim$ Bap $\in \mu^{*}$

From (9) by (C.B*) 
(10) and (11) violate (C. $\sim$ ), thus reducing the counterassumption ad absurdum. The proof in $\mathrm{H}^{*}$ shows that Hintikka's argument alone does not justify the inclusion of axiom (5) in a logic of belief. In the next section I will argue that there are independent reasons to reject (5) and to adopt the weaker set of axioms.

The conditions that Hintikka imposes on model sets also differ from the ones in our system. Instead of our condition (C.CB), Hintikka includes the following condition in his system:

(C.BB*) If $B_{a} p \in \mu$ and if $\mu *$ is an alternative to $\mu$ in some model system $\Omega$, then $B_{a} p \in \mu *$.

Notice the difference between (C.BB*) and (C.CB). The former says that if I believe something in $\mu$, I believe it in every alternative to $\mu$. The latter says that if I believe something in $\mu$, there is at least one alternative to $\mu$ in which I believe it. (C.BB*) entails (C.CB), but not the converse. Intuitively, if I believe that $p$ in $\mu$, (C.CB) does not rule out the possibility of there being alternatives to $\mu$ in which I do not believe that $p$. This is not a problem, for all that is needed for my beliefs to be consistent is that there be at least one alternative model set in which they are true. If I believe that $p$, (C.B) alone guarantees that there is at least one alternative model set in which $p$ is true. (C.CB) is added to reflect our intuition that there are some model sets in which $p$ is true and I believe it, and others in which $p$ is true and I do not believe it.

The difference between (C.CB) and (C.BB*), of course, simply reflects our choice of axiom A3 instead of Hintikka's axiom (5). In our system, the sentence

$$
B_{a} p \supset C_{a} \sim B_{a} p
$$


is not a contradiction. If $B_{a} p \in \mu$, there may be an alternative $\mu *$ to $\mu$ such that $\sim B_{a} p \in \mu *$. Hence, $B_{a} p \supset B_{a} B_{a} p-$ Hintikka's axiom (5)—will be false in some model sets. On the other hand, according to our condition (C.CB), if $B_{a} p$ $\in \mu$, then there is at least one alternative $\mu *$ to $\mu$ such that $B_{a} p \in \mu *$. Therefore, our third axiom, $B_{a} p \supset C_{a} B_{a} p$, is true in every model set of our system. In the remaining sections of the essay I will provide further reasons to support the claim that Hintikka's axiom (5) should not be a theorem in a logic of belief and that it should be replaced by axiom A3.

\section{Believing That ONE Believes}

Prima facie, Hintikka's axiom (5) seems extremely plausible. If I believe that $p$, it seems absurd to deny that I believe that I believe that $p$. But the obviousness of (5) disappears when the sentence is not in the first-person. It is not absurd to assert of someone else that he believes that $p$ but he does not believe that he believes that $p$. Consider the truth conditions for the sentence $B_{a} p$. The sentence is true iff $a$ is in an intentional state whose content is $p$ and whose propositional attitude is belief. $\sim B_{a} p$, on the other hand, is true iff $a$ is not in that intentional state. Now consider the truth conditions for the sentence $B_{a} B_{a} p$. The sentence is true iff $a$ is in an intentional state whose content is $B_{a} p$ and whose mental attitude is belief. In other words, $B_{a} B_{a} p$ is true iff $a$ 's belief that $p$ is accompanied by a concurrent belief whose content is $B_{a} p$. The negation of $B_{a} B_{a} p$, on the other hand, is true iff $a$ is not in the intentional state of believing that $B_{a} p$.

It is perfectly possible that when someone believes that $p$, no concurrent belief occurs about that person's belief that $p$. For example, the sentence 
(13) 'Mary believes that it is raining but she does not believe that she believes that it is raining'

is not absurd or self-contradictory. A virtue of our system is that it captures this important fact about the notion of belief. The sentence

$$
B_{a} p \& \sim B_{a} B_{a} p
$$

is not a contradiction in $\mathrm{H}^{*}$. But notice that $\left(13^{*}\right)$ is simply the negation of Hintikka's axiom (5). If we accept (5) as an axiom, we would also have to claim that (13) and $\left(13^{*}\right)$ are contradictions (which is clearly not the case). Therefore, unless we want to misrepresent an important aspect of the analysis of the notion of belief, (5) cannot be an axiom of the system.

What, then, about the absurdity of uttering sentence $\left(13^{*}\right)$ when $a$ is the speaker? One option is to modify our system and include an ad hoc clause about the performance conditions of sentences in the first person. ${ }^{3}$ But there is no need to do so. Notice that $\left(13^{*}\right)$ is just a version of Moore's Paradox and can be treated as such. In order to examine the oddity of $\left(13^{*}\right)$, we must now turn to the analysis of Moore's Paradox.

${ }^{3}$ Axiom (5) makes Hintikka's system a KD4 system of epistemic logic. Rieger (2015) shows that adding the negation of a sentence that states Moore's Paradox to a KD system would be sufficient to block it, but that move seems equally ad hoc. The present proposal is based on a revision of the modal operator $C_{a}$ itself, which leads directly to a rejection of Axiom (5).

Manuscrito - Rev. Int. Fil. Campinas, v. 43, n. 2, pp. 1-15, Abr.-Jun. 2020. 


\section{EXPLAINING THE PARADOX}

Before considering the problem described above, I will return to the original paradox in order to show that Hintikka's proposed solution is also valid in our weaker system. We can symbolize Moore's Paradox in the following terms:

(1*) $\quad p \& \sim B_{a} p$.

The sentence is not a contradiction because

(14) $\quad p \supset B_{a p}$

is not a theorem in either system. Hintikka's ingenious solution to the paradox is to argue that although $\left(1^{*}\right)$ is not a contradiction, the following sentence is:

$$
B_{a}\left(p \& \sim B_{a} p\right)
$$

This sentence corresponds to the general presumption that the speaker believes or at least can conceivably believe what he or she says. In Hintikka's words, "the gist of Moore's Paradox may be said (somewhat elliptically) to lie in the fact that $[(15)]$ is necessarily unbelievable by the speaker" (p. 67). A virtue of this approach is that nothing turns on the peculiarities of the first-person singular pronoun. (15) is contradictory no matter who $a$ is.

Instead of presenting Hintikka's proof that (15) is a contradiction, I will present a similar proof in $\mathrm{H}^{*}$, and indicate the pertinent differences. The proof is a reductio of the following counterassumption:

(17) $\quad p \& \sim B_{a} p \in \mu^{*}$

Counterassumption

From (16) by (C.B*)

Manuscrito - Rev. Int. Fil. Campinas, v. 43, n. 2, pp. 1-15, Abr.Jun. 2020. 


$$
\begin{aligned}
& B_{a}\left(p \& \sim B_{a} p\right) \in \mu^{*} \quad \text { From (16) by (C.CB) } \\
& \text { (19) } \sim B_{a} p \in \mu^{*} \quad \text { From (17) by (C.\&) } \\
& \text { (20) } \left.C_{a} \sim p \in \mu * \quad \text { From (19) by (C.B } \mathrm{B}_{\mathrm{Def}}\right) \\
& \text { (21) } \sim p \in \mu * * \quad \text { From (20) by (C.C) } \\
& \text { (22) } p \& \sim B_{a} p \in \mu * * \quad \text { From (18) by (C.B*) } \\
& \text { (23) } p \in \mu^{* *} \quad \text { From (22) by (C.\&) }
\end{aligned}
$$

Here (21) and (23) contradict (C. $\sim$ ), thus completing the reductive argument. The main difference between our argument and Hintikka's is the justification of (18). Instead of (C.CB), which is not a condition in Hintikka's system, he uses $\left(\mathrm{C} . \mathrm{BB}^{*}\right)$. This difference is unimportant because, as Hintikka admits at one point, the proof could be done without making use of that condition.

We can now return to the version of Moore's Paradox presented in the previous section. Following Hintikka's strategy, we can prove that although $\left(13^{*}\right)$ is not a contradiction in $\mathrm{H}^{*}$, the following sentence is:

$$
B_{a}\left(B_{a} p \& \sim B_{a} B_{a} p\right)
$$

The proof is a reductio of the following counterassumption:

$\begin{array}{lll}\text { (25) } & B_{a}\left(B_{a} p \& \sim B_{a} B_{a} p\right) \in \mu & \text { Counterassumption } \\ \text { (26) } & B_{a} p \& \sim B_{a} B_{a} p \in \mu^{*} & \text { From (25) by (C.B }) \\ (27) & B_{a}\left(B_{a} p \& \sim B_{a} B_{a} p\right) \in \mu^{*} & \text { From (25) by (C.CB) } \\ (28) & \sim B_{a} B_{a} p \in \mu^{*} & \text { From (26) by (C.\&) } \\ (29) & C_{a} \sim B_{a} p \in \mu^{*} & \text { From (28) by (C.B } \text { Def }) \\ (30) & \sim B_{a} p \in \mu^{* *} & \text { From (29) by (C. C) } \\ (31) & B_{a} p \& \sim B_{a} B_{a} p \in \mu^{* *} & \text { From (27) by (C.B*) } \\ \text { (32) } & B_{a} p \in \mu^{* *} & \text { From (31) by (C. } \&)\end{array}$


(30) and (32) contradict (C. $\sim$ ), thus completing the reductive argument. Just as in the original version of the paradox, (24) is a contradiction regardless of the identity of $a$.

This analysis of $\left(13^{*}\right)$ explains the problematic case in which the sentence is in the first-person and gives further plausibility to my choice of axiom A3. In fairness to Hintikka, I must admit that the advantages of my system over the one he developed in Knowledge and Belief depend entirely on my reinterpretation of the modal operator $C_{a}$. I believe, however, that this reinterpretation, and the system that can be constructed on it, give us a better picture of the logical structure of sentences containing the notion of belief.

\section{CONCLUSION}

Epistemic logic has come a long way since the publication of Hintikka's seminal work, and there are other ways of dissolving and explaining the paradox in the literature 4 . My purpose in this paper, however, was merely historical. I wanted to show how it is possible to modify Hintikka's original axioms and definitions to provide a system that is better attuned to our philosophical intuitions. In particular, I presented a better interpretation of the modal operator $C_{a}$, which leads directly to a rejection of the idea that when someone believes that $p$, there must be a concurrent belief about that person's belief that $p$. More formally, $B_{a} p \&$ $\sim B_{a} B_{a} p$ is not a contradiction in $\mathrm{H}^{*}$. This formula is the negation of axiom (5) in Hintikka's original system. The formula is also a version of Moore's Paradox when $a$ is the speaker. As I show in the last section, it can be established in $\mathrm{H}^{*}$ that $B_{a}\left(B_{a} p \& \sim B_{a} B_{a} p\right)$ is a contradiction, so Hintikka's original explanation of the paradox remains valid in the new

${ }^{4}$ See Green and Williams (2007) for a survey.

Manuscrito - Rev. Int. Fil. Campinas, v. 43, n. 2, pp. 1-15, Abr.Jun. 2020. 
system. It remains to be seen how the system $\mathrm{H}^{*}$ relates to Hintikka's analysis of knowledge, but that is left for future work.

\section{REFERENCES}

Hintikka, J. (1962). Knowledge and belief: An introduction to the logic of the two notions. Ithaca: Cornell University Press.

Green, M. S., \& Williams, J. N. (Eds.) (2007). Moore's Paradox: New essays on belief, rationality and the first-person. New York: Oxford University Press.

Moore, G. E. (1942). A reply to my critics. In P. A. Shilpp (Ed.), The philosophy of G. E. Moore. New York: Tudor Publishing Company.

(1944). Russell's 'theory of descriptions'. In P. A. Shilpp (Ed.), The philosophy of Bertrand Russell. New York: Tudor Publishing Company.

Rieger, A. (2015). Moore's paradox, introspection and doxastic logic. Thought, 4, 215-227.

Searle, J. (1969). Speech acts: An essay in the philosophy of language. Cambridge: Cambridge University Press.

$\mathrm{BY}$ 